\title{
Editorial
}

Pathobiology

Pathobiology 2011;78:293-294

DOI: $10.1159 / 000331226$

\section{Future Perspectives of Gastric Cancer Treatment - From Bench to Bedside}

Cancer, a chronic proliferative disease with multiple genetic and epigenetic alterations, develops as a result of an accumulation of various endogenous and exogenous causes. Recent advances in cancer research have uncovered the molecular mechanisms of the development and progression of the disease. Multiple alterations during carcinogenesis are found in tumor suppressor genes, oncogenes, DNA repair genes, cell cycle regulators, cell adhesion molecules, growth factors/receptors, matrix metalloproteinases and other sites. Because of different but also common molecular bases, each cancer displays a different biological behavior and response to treatment. Recent focus has been on the presence of cancer stem cells in terms of chemoradiotherapy.

Amongst 10 million people diagnosed with cancer in the world, the most common cancers are those of the lung, breast and stomach. However, there are marked regional differences in the organs that are affected. In eastern Asia including Japan, Korea and China, cancers of the stomach, lung, liver and esophagus are of major concern, although cancers of the breast and colorectum are also increasing. Mortality due to gastric cancer, one of the most common cancers worldwide, is second to lung cancer. The highest rates of gastric cancer occur in eastern Asia, South America and Eastern Europe. Of particular note, more than half of the gastric cancer in the world occurs in Japan, China and Korea. Advances in diagnosis and treatment have enabled us to offer excellent longterm survival for early cancer, but the prognosis for advanced cancer still remains poor. The International Gas- tric Cancer Association, founded in 1995, contributes greatly to research into the carcinogenesis, diagnosis and treatment of gastric cancer; a congress on these issues was held recently, in 2011, in Seoul, Korea. Many scientific events on gastric cancer have been organized in these countries, such as the international symposium 'Future Perspectives of Gastrointestinal Cancer Treatment From Bench to Bedside' at the occasion of the 20th Annual Meeting of the Japanese Society for Gastroenterological Carcinogenesis, the 17th Seoul International Cancer Symposium 'Gastric Cancer Update 2010', among others.

In this issue of Pathobiology, distinguished experts in Asia review current topics and future perspectives of gastric cancer treatment in molecular pathology, tissue engineering, surgery and chemotherapy. Woo et al. present the biological significance of STAT3 activation in gastric cancer. They analyzed a large number of gastric cancer specimens on tissue arrays immunohistochemically using several antibodies including anti-phospho-Tyr705STAT3, an active form of STAT3. Nuclear STAT3 activation was an early event in carcinogenesis and significantly correlated with better prognosis, proliferation and HIF- $1 \alpha$ activation in gastric cancer, suggesting that the nuclear PSTAT3 may serve as a valuable prognostic factor and therapeutic target in gastric carcinoma. Jang and Kim review the recent understanding of the molecular pathology of gastric cancer. Gastric cancer develops through multistep processes that begin with Helicobacter pylori-induced atrophic gastritis. Genetic and epigenetic

\begin{tabular}{ll}
\hline KARGER & (c) 2011 S. Karger AG, Basel \\
1015-2008/11/0786-0293\$38.00/0 \\
$\begin{array}{l}\text { Fax +41 61 306 1234 } \\
\begin{array}{l}\text { E-Mail karger@karger.ch } \\
\text { www.karger.com }\end{array}\end{array}$ & $\begin{array}{l}\text { Accessible online at: } \\
\text { www.karger.com/pat }\end{array}$
\end{tabular}


alterations include TP53, CTNNB1 and Runx3. Gastric cancer with microsatellite instability is a well-defined subset exhibiting distinctive clinicopathological features. Targeted therapy using trastuzumab against gastric cancer with ERBB2/HER2 amplification shows a better prognosis. A genome-wide search to identify novel methylation-silenced genes will provide novel opportunities in the treatment of gastric cancer. For the treatment of early cancer of the upper gastrointestinal tract, endoscopic submucosal dissection is widely used. Takagi et al. describe the usefulness of autologous oral mucosal epithelial cell sheets to prevent stenosis after esophageal endoscopic submucosal dissection. Human oral mucosal epithelial cells can be successfully cultured and harvested as continuous cell sheets without any animal-derived materials. The method for fabricating epithelial cell sheets shown here is suitable for the validation for clinical trials. Yang describes the importance of translational research in the clinical field and of answering the questions of the operating room by basic research. The clinical questions include the source of disseminated cancer cells during gastric surgery, the implications of serum gastrin level after proximal gastrectomy and the exploration of local drug delivery systems for gastric cancer. Various concepts and technologies, such as epigenetics, microRNA, genetic polymorphism and the microarray technique, have contributed to uncovering molecular stomach carcinogenesis. The next generation of sequencing is a promising revolutionary tool. Isogaki et al. discuss the outline of robot-assisted surgery for gastric cancer. The da Vinci surgical system was developed for minimally invasive surgery. While the procedures of the gastrectomy are similar to those of the usual laparoscopic surgery, several aspects such as the port placement and the role of the assistant are modified. Robot-assisted gastrectomy using the da Vinci system can be applied safely and effectively for the treatment of advanced gastric cancer even with lymph node dissection. Higuchi et al. review the current status of chemotherapy for gastric cancer and discuss future perspectives. Numerous randomized trials of various chemotherapeutic regimens have contributed to improved outcomes in patients with advanced gastric cancer. The standard regimen for advanced gastric cancer in Japan is a combination of S- 1 and cisplatin. Recently, new drug development has focused on molecular target agents, and personalized therapy for advanced gastric cancer has just begun. Since abundant information about the heterogeneity and biological background of gastric cancer has been compiled, new strategies for personalized therapy will be introduced in the future. Yoshida et al. describe the roles of surgical oncologists in the new era. Surgical oncologists have two main roles in the treatment of gastric cancer. One is to provide minimally invasive surgery for early gastric cancer patients and the new concept of surgical intervention for advanced and metastatic disease. The second is to evaluate the significant values of the aggressive treatment, called 'adjuvant surgery', for stage IV gastric cancer patients who have successfully responded to initial chemotherapy for curative intent.

In conclusion, this issue of Pathobiology presents reviews of the current status and the future perspectives of the pathology, research and treatment of gastric cancer. A detailed understanding of the molecular pathogenesis and individual character of gastric cancer will contribute to providing personalized cancer care. The new development of medical technologies and therapeutic agents will enable us to provide the best treatment for patients of both early and advanced cancer. The viewpoints from bench to bedside and clinic to bench are absolutely crucial.

Wataru Yasui 